\title{
Theory of percolation and tunneling regimes in nanogranular metal films
}

\author{
Claudio Grimaldi \\ Laboratory of Physics of Complex Matter, Ecole Polytechnique Fédérale de Lausanne, Station 3, CP-1015 Lausanne, Switzerland
}

\begin{abstract}
Nanogranular metal composites, consisting of immiscible metallic and insulating phases deposited on a substrate, are characterized by two distinct electronic transport regimes depending on the relative amount of the metallic phase. At sufficiently large metallic loadings, granular metals behave as percolating systems with a well-defined critical concentration above which macroscopic clusters of physically connected conductive particles span the entire sample. Below the critical loading, granular metal films are in the dielectric regime, where current can flow throughout the composite only via hopping or tunneling processes between isolated nanosized particles or clusters. In this case transport is intrinsically non-percolative in the sense that no critical concentration can be identified for the onset of transport. It is shown here that, although being very different in nature, these two regimes can be described by treating percolation and hopping on equal footing. By considering general features of the microstructure and of the electrical connectedness, the concentration dependence of the dc conductivity of several nanogranular metal films is reproduced to high accuracy within an effective medium approach. In particular, fits to published experimental data enable us to extract the values of microscopic parameters that govern the percolation and tunneling regimes, explaining thus the transport properties observed in nanogranular metal films.
\end{abstract}

PACS numbers: 73.40.Gk, 64.60.ah, 72.80.Tm, 81.05.Rm

\section{INTRODUCTION}

Among the different classes of conductor-insulator composites, nanogranular metal films are quite unique materials as they display distinct and tunable electrical, optical, and magnetic properties depending on the nature and concentration of the metallic phase, as well as on the structure of the films $\underline{-3}$ In the preparation of this class of composites, various sputtering, evaporation, and ion implantation methods are used to deposit immiscible metals and insulators on a substrate to form composite films with a wide range of the volume fraction $\phi$ of the metallic phase. At large $\phi$, the composite is basically a metallic continuum whose electronic conductivity $\sigma$ is limited by grain boundaries and scattering with few insulating inclusions. As $\phi$ decreases, $\sigma$ is lowered by the enhanced concentration of the insulating phase. This "metallic regime" persists until matrix inversion occurs at a material dependent critical value $\phi_{c}$, below which the metallic continuum is broken up into disconnected metallic particles or clusters dispersed in the insulating phase. In this "dielectric regime", electrons flow throughout the composite only by tunneling or hopping processes between isolated, and homogeneously dispersed, nanometric metallic particles.

These two distinct, structurally driven, transport regimes are very different in nature, as seen from the temperature and relative concentration dependences of $\sigma$. Above $\phi_{c}$, granular metal films are assimilable to percolative systems in which coalescing metallic particles form a system spanning conductive network. As a function of $\phi$, the resulting conductivity for $\phi \gtrsim \phi_{c}$ is thus expected to follow a percolation power-law behavior of the form: $\underline{\underline{4}}, \underline{\underline{ }}$

$$
\sigma \simeq \sigma_{0}\left(\phi-\phi_{c}\right)^{t}
$$

where $\sigma_{0}$ is a constant and $t \simeq 2(t \simeq 1.3)$ is the universal transport exponent for three-dimensional (twodimensional) systems. Furthermore, for $\phi>\phi_{c}$ transport shows typically a metallic behavior, with the resistivity increasing linearly with the temperature. In contrast to the percolation mechanism implied by Eq. (1), tunneling between submicron conducting particles in the dielectric regime hints to the absence of any "critical" concentration, as electrons have to tunnel across interparticle distances that increase gradually as $\phi$ is reduced. Considering that in the limit of dilute particles of size $D$ the mean particle separation $\delta$ scales as $\delta \propto D / \phi^{1 / d}$, where $d$ is the system dimensionality, the tunneling conductivity $\sigma \propto \exp (-2 \delta / \xi)$ for sufficiently large temperatures is thus expected to follow:

$$
\sigma \propto \exp \left(-a_{d} \frac{D}{\xi \phi^{1 / d}}\right),
$$

where $\xi$ is the tunneling decay length and $a_{d}$ is a dimensionless constant, which for point particles dispersed in a three-dimensional (two-dimensional) volume takes up the value $a_{3} \simeq 1.41\left(a_{2} \simeq 2.12\right) \stackrel{6.7}{ }$ As a function of temperature $T$, the dielectric regime is associated with a stretched exponential behavior of the form:

$$
\sigma \propto \exp \left(-\sqrt{\frac{T_{0}}{T}}\right),
$$

which arises from tunneling processes in the presence of a Coulomb gap. ${ }^{8-11}$ Equation (3) applies for temperatures lower than a $\phi$-dependent characteristic temperature, $T_{0}$, which typically increases from $T_{0} \sim 100 \mathrm{~K}$ for $\phi \lesssim \phi_{c}$ to a few thousands of Kelvin for $\phi$ values deep in the dielectric region. 1,12

The limiting $\phi$-dependencies of $\sigma$ highlighted in Eqs. (11) and (2) arise from general considerations which do not rely on the detailed knowledge of the composite 
film morphology. However, while Eq. (1) is shown to properly fit the measured $\sigma$ in the metallic regime, $\underline{13-17}$ the exponential behavior of Eq. (2) is less often used to interpret the observed $\phi$-dependence of the dielectric regime $\stackrel{15,18}{ }$ In Ref. 14, for example, the dielectric region is understood in terms of Eq. (11) with a tunnelinginduced nonuniversal exponent,$\frac{19}{1}$ while in Refs. 15 and 16 additional percolation transitions are considered to be active in the $\phi<\phi_{c}$ region. The power law behavior of Eq. (11), or its generalizations, requires however either a cut-off in the inter-particle conductances $\stackrel{20}{\underline{20}}$ or very peculiar (crystal-like) arrangements of the metallic particles in the matrix $\stackrel{21}{=}$ both of which are difficult to justify from the disordered morphology of nanogranular films in the dielectric regime. Furthermore, the variable range hopping mechanism at the basis of the stretched exponential behavior of Eq. (3) is, in principle, incompatible with the notion of a fixed cut-off distance between the particles. From these considerations, we see that although the temperature dependence of $\sigma$ is quite well understood,,$\underline{10}$ there is still no general consensus on how to interpret the behavior of the conductivity as a function of the metallic content at fixed temperatures. In particular, there is a need to further understand the different regimes of granular metals within a single, coherent, description.

In this paper, we present an effective medium formulation that naturally accounts for the metallic (percolation) and dielectric (tunneling) regimes of granular thick films, and the transition between them. With the term "thick film" we mean that the film thickness is much larger than the typical particle size, so that the system is three dimensional. By considering general properties of the microstructure and of the electrical connectedness, we clarify how the percolation behavior of Eq. (1) for $\phi>\phi_{c}$ evolves into the exponential one of Eq. (2) for $\phi<\phi_{c}$. In this way, we can reproduce the room temperature conductivity data of several composite films in the whole range of $\phi$, and extract from experiments the tunneling characteristics, the percolation threshold, and the microscopic conductances governing the overall conductivity behavior. Furthermore, by using a cherry-pit model for the conductive particle dispersion in the films, we identify the different observed values of $\phi_{c}$ in terms of partial overlaps between the particles, providing thus a simple microscopic interpretation for the location of the dielectric-metallic transition.

\section{MODEL AND EFFECTIVE MEDIUM APPROXIMATION}

During the deposition process of granular metal films, the metallic particles nucleate and grow giving rise to a spatial distribution of the metallic grains which depends on the relative amount of metallic and insulating phases, on the interaction between them, and on the film growth conditions. Detailed description of film microstructure requires thus specific knowledge of the mi-

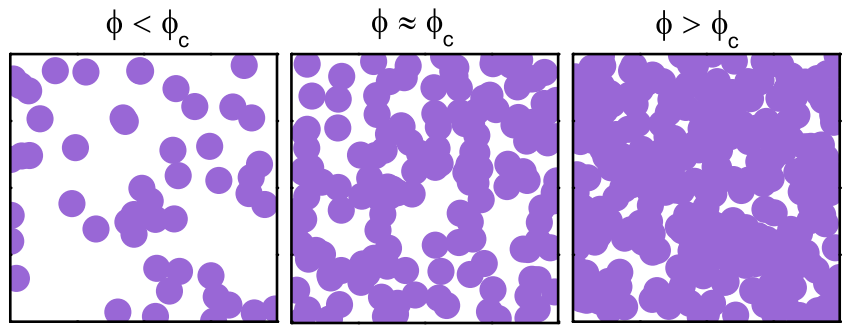

(a)

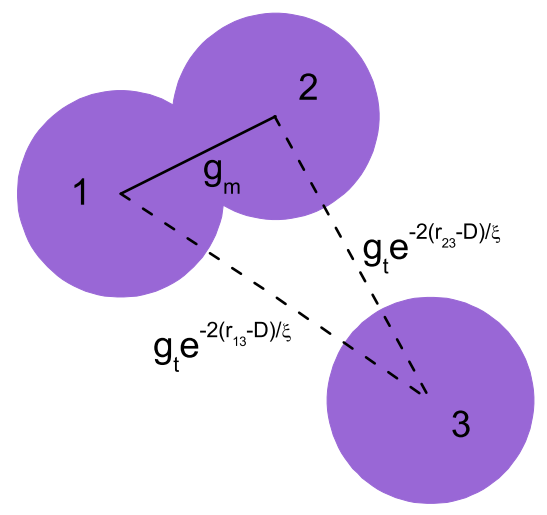

FIG. 1. (Color online) (a) Illustration of the model for nanogranular composite films in which the metallic particles are represented as partially overlapping spheres dispersed in a continuum matrix. For volume fractions $\phi$ lower than the percolation threshold $\phi_{c}$, the composite is constituted by dispersions of isolated particles and clusters of overlapping particles. For $\phi>\phi_{c}$ the film microstructure is modeled as a continuum of overlapping spheres with isolated voids. $\phi_{c}$ is the critical volume fraction for the percolation of overlapping spheres. (b) Model of inter-particle conductances. For any two overlapping spheres, the conductance is set equal to $g_{m}$, as for spheres 1 and 2. When two particles do not overlap, their conductance is chosen to be of tunneling type, as for spheres 1 and 3 , and 2 and 3 .

croscopic processes governing the spatial distribution of the two phases. However, observational studies of many different film microstructures evidence quite general features, such as homogeneity and disorder of particle dispersions, nanometric size of metallic grains in the dielectric regime, and matrix inversion in the transition region. These generic features can be taken into account without detailed knowledge of the processes governing them by employing a minimal model of the microstructure chosen as to capture the essential aspects.

To define a minimal model for granular metal films we consider spherical metallic particles with equal diameter $D$ dispersed in a continuum insulating medium. We simulate coalescing between the metallic particles by allowing the spheres to overlap to some extent. At low volume fractions, the metallic phase is thus composed primarily of isolated spheres, while at large $\phi$ the composite consists basically of a metallic continuum with few 
isolated voids, as shown schematically in Fig. 1(a). The regions of low and large $\phi$ correspond respectively to the dielectric and metallic regimes of the granular films. The critical volume fraction $\phi_{c}$ separating these two regions corresponds to the geometrical percolation threshold for intersecting spheres, i.e., $\phi_{c}$ is the smallest $\phi$ such that a system spanning cluster of overlapping spheres exists. The specific value of $\phi_{c}$ depends on the degree of particle overlapping and on the statistical properties of the dispersion, which however we do not specify at the moment.

To model the electrical connectedness at the microscopic level, we define two kinds of interparticle conductances, as illustrated schematically in Fig. 1(b). When two particles overlap, as for example particles 1 and 2 in Fig. 1(a), we assume that the interparticle conductance is constant and independent of the degree of overlapping. Between nonoverlapping particles, instead, we ascribe a tunneling conductance which decays exponentially with the relative distance between the particles, as for the pairs of spheres 1,3 and 2,3 in Fig. 1(b). For any two given spheres $i$ and $j$, the interparticle conductance assumes thus the following form:

$$
g_{i j}= \begin{cases}g_{m} & \text { for } r_{i j} \leq D, \\ g_{t} e^{-2\left(r_{i j}-D\right) / \xi} & \text { for } r_{i j}>D,\end{cases}
$$

where $r_{i j}$ is the distance between the sphere centers and $\xi$ is the tunneling decay length. The prefactors $g_{m}$ and $g_{t}$ in Eq. (4) are in general different: $g_{t}$ is in principle smaller or much smaller than the conductance of two coalesced particles as the electron has to cross an interfacial barrier even if two particles are at contact. Furthermore, for the case of nanosized ferromagnetic particles, $g_{t}$ depends also on the relative spin polarization $\underline{\underline{22}}$

In writing Eq. (4) we assume that particle charging and Coulomb interaction effects do not appreciably contribute to the exponential decay for $r_{i j}>D$. Although strictly valid for infinite temperatures, this approximation is nevertheless justified for granular metals at room temperature and with metallic contents not much below $\phi_{c}$, as in this case the variable range hopping characteristic temperature in Eq. (3) is typically $T_{0} \sim 100 \mathrm{~K}, \underline{12}$ Since we are interested in the $\phi$-dependence of $\sigma$ at room temperature, we can think of the prefactor $g_{t}$ as to partially include particle charging and Coulomb interactions.

\section{A. Effective medium approximation}

We proceed to evaluating the composite film conductivity by using an effective medium approximation (EMA) previously applied successfully to a number of different conductor-insulator composites $\underline{21,23-26}$ A detailed derivation of EMA is presented in Ref. 23. Here we describe a simple method to derive the EMA equation within the two-site approximation by considering a system of $N$ metallic spheres dispersed in a volume $V$. We then construct a resistor network whose node positions coincide with the centers of the spherical particles. The corresponding bond conductances are given by the set of $N(N-1) / 2$ conductances of Eq. (4). This network is complete, which means that to each pair of nodes is associated a finite conductance $g_{i j}$. The two-point resistance $R_{i j}$ between any two nodes $i$ and $j$ is thus a well defined quantity, from which we construct the average resistance of the network:

$$
\langle R\rangle=\frac{1}{N(N-1)}\left\langle\sum_{i, j}{ }^{\prime} R_{i j}\right\rangle,
$$

where $\langle\cdots\rangle$ indicates a configurational average and the prime symbol means that the term with $i=j$ is omitted from the summation. We can express $R_{i j}$ as given by the direct resistance between $i$ and $j$, i.e., $1 / g_{i j}$, in parallel with the resistance $1 / G_{i j}^{\prime}$ of a network in which $g_{i j}$ has been removed from the system:

$$
R_{i j}=\frac{1}{g_{i j}+G_{i j}^{\prime}} .
$$

Next, we introduce a second (effective) network, with spatial distribution of nodes identical to the original one, in which the conductances are all identically equal to $\bar{g}$, independently of the node indexes. This second network is a complete network whose two-point resistance is simply given by $\bar{R}=1 / \bar{G}=2 / N \bar{g} \stackrel{27}{2}$ We want to find $\bar{g}$ such that the resistance difference between the two networks

$$
\langle R\rangle-\bar{R}=\frac{1}{N(N-1)}\left\langle\sum_{i, j}^{\prime}\left(\frac{1}{g_{i j}+G_{i j}^{\prime}}-\frac{2}{N \bar{g}}\right)\right\rangle
$$

vanishes. To this end, we apply the two-site EMA which amounts to replacing $G_{i j}^{\prime}$ by the two-point conductance of the effective network minus the direct contribution between $i$ and $j$ :

$$
G_{i j}^{\prime} \rightarrow \bar{G}-\bar{g}=(N / 2-1) \bar{g},
$$

so that Eq. (7) reduces to:

$$
\frac{\langle R\rangle-\bar{R}}{\bar{R}}=\frac{1}{N(N-1)}\left\langle\sum_{i, j}^{\prime} \frac{\bar{g}-g_{i j}}{g_{i j}+(N / 2-1) \bar{g}}\right\rangle .
$$

Imposing $\langle R\rangle=\bar{R}$ to the above expression, after some algebra and setting $N \gg 1$ we find the following equation for the effective conductance $\bar{G}, 21,23$

$$
\frac{1}{N}\left\langle\sum_{i, j}^{\prime} \frac{g_{i j}}{g_{i j}+\bar{G}}\right\rangle=2 .
$$

Since the conductances $g_{i j}$ in Eq. (4) depend only upon the relative distances $r_{i j}$, we can replace the summation over $i, j$ by an integral over the continuous distance $r: \underline{21}, 23$ By using Eq. (4) we thus obtain for three dimensional systems:

$$
Z(\phi, D) \frac{g_{m}}{\bar{G}+g_{m}}+4 \pi \rho \int_{D}^{\infty} d r r^{2} g_{2}(r) \frac{g_{t} e^{-\frac{2(r-D)}{\xi}}}{\bar{G}+g_{t} e^{-\frac{2(r-D)}{\xi}}}=2,
$$


where

$$
g_{2}(r)=\int \frac{d \Omega}{4 \pi}\left\langle\frac{1}{N \rho} \sum_{i, j}{ }^{\prime} \delta\left(\mathbf{r}-\mathbf{r}_{i j}\right)\right\rangle
$$

is the radial distribution function for the conducting spheres, $\stackrel{28}{2}$ and

$$
Z(\phi, D)=4 \pi \rho \int_{0}^{D} d r r^{2} g_{2}(r)
$$

is the coordination number for intersecting spheres, which measures how many spheres on average overlap a given sphere for a given concentration 29 Finally, $\rho=$ $N / V$ is the particle number density which, depending on the degree of sphere overlapping, determines the fractional coverage $\phi$ of the metallic phase.

\section{B. EMA dielectric and metallic regimes}

Equations (11) and (13) enable us to relate the behavior of the overall transport with the morphology of the composite through the radial distribution function $g_{2}(r)$, once this is known. Detailed knowledge of $g_{2}(r)$ is however not necessary to extract some important limiting behaviors of $\bar{G}$ from the solution of Eq. (11). For example, the EMA dielectric regime is obtained by noticing that in the dilute limit $\phi \ll 1$ the metallic particles are uncorrelated $\left[g_{2}(r) \simeq 1\right]$ and practically do not overlap $[Z(\phi, D) \ll 1]$. In this way, the first term in the left-hand side of Eq.(11) can be neglected and the EMA equation reduces to:

$$
\frac{24 \phi}{D^{3}} \int_{D}^{\infty} d r r^{2} \frac{g_{t} e^{-\frac{2(r-D)}{\xi}}}{\bar{G}+g_{t} e^{-\frac{2(r-D)}{\xi}}}=2
$$

where we have set $\phi \simeq \pi \rho D^{3} / 6$. The above integral is exactly solvable and the left-hand side of Eq. (14), which we denote by $I$, can be expressed in terms of polylogarithm functions. We find it more practical, however, to use for $I$ the following approximation which is very accurate for all values of $\bar{G}$ :

$$
I=8 \phi\left\{\left[1+\frac{\xi}{2 D} \ln \left(\frac{g_{t}+\bar{G}}{\bar{G}}\right)\right]^{3}-1\right\} .
$$

From $I=2$ we thus find for small $\phi$ :

$$
\begin{aligned}
\bar{G} & \simeq g_{t} \exp \left\{-\frac{2 D}{\xi}\left[\left(\frac{1}{4 \phi}+1\right)^{1 / 3}-1\right]\right\} \\
& \underset{\phi \rightarrow 0}{\longrightarrow} g_{t} \exp \left(-1.26 \frac{D}{\xi \phi^{1 / 3}}\right),
\end{aligned}
$$

which has the same asymptotic behavior of Eq. (2). Equation (16) can also be recovered from the method described in Ref. 21.
To obtain the EMA version for the percolating regime, we neglect the tunneling contributions in Eq. (11). The EMA equation reduces to $Z(\phi, D) g_{m} /\left(\bar{G}+g_{m}\right)=2$, from which we find:

$$
\bar{G}=g_{m}\left[\frac{Z(\phi, D)}{2}-1\right]
$$

Since $Z(\phi, D)$ increases monotonically with $\phi, \bar{G}$ is nonnegative only for $\phi \geq \phi_{c}$, where $\phi_{c}$ satisfies $Z\left(\phi_{c}, D\right)=2$. Hence, by expanding Eq. (17) in the vicinity of $\phi_{c}$ we obtain for $\phi-\phi_{c} \gtrsim 0$ :

$$
\bar{G} \simeq \frac{g_{m} Z^{\prime}\left(\phi_{c}, D\right)}{2}\left(\phi-\phi_{c}\right)
$$

which is the EMA equivalent of the percolation conductivity of Eq. (1), in which the transport exponent is unity rather than $t \simeq 2$.

\section{MINIMAL EMA MODEL FOR NANOGRANULAR METAL FILMS}

From the results of the previous section, we are now in the position of formulating a minimal, phenomenological model describing the $\phi$ dependence of the conductivity of nanogranular metal films. The starting point is Eq. (11), which we modify in the following way. First, motivated by the observation that the microstructure of the film is expected to have little influence in the dilute particle limit $\phi \ll 1$, where tunneling dominates, we replace the second term in the left-hand side of (11) with Eq. (15). Next, to keep the number of independent parameters to an absolute minimum, we assume a simple linear dependence of the coordination number: $Z(\phi, D)=b \phi$. In this way, the critical volume fraction is uniquely identified by $\phi_{c}=2 / b$, as can be verified by using Eq. (17). Finally, to recover the correct exponent in the percolating regime, we follow the phenomenological approach of Ref. 30 and replace the quantity $g_{m} /\left(\bar{G}+g_{m}\right)$ in Eq. (11) with $g_{m}^{1 / t} /\left(\bar{G}^{1 / t}+g_{m}^{1 / t}\right)$, where we set $t=2$ for three dimensional materials. The resulting EMA equation reduces thus to:

$$
\frac{\left(\phi / \phi_{c}\right) g_{m}^{1 / t}}{\bar{G}^{1 / t}+g_{m}^{1 / t}}+4 \phi\left\{\left[1+\frac{\xi}{2 D} \ln \left(\frac{g_{t}+\bar{G}}{\bar{G}}\right)\right]^{3}-1\right\}=1
$$

It is easy to see from the above equation that for $\phi \ll \phi_{c}$ the EMA conductance reduces to Eq. (16), while for $\phi \gtrsim \phi_{c}$ (and for sufficiently small $\left.\xi / D\right)$ it takes the percolation form $\bar{G} \propto\left(\phi-\phi_{c}\right)^{t}$. It is worth stressing that while the tunneling contribution is treated explicitly, the percolation threshold is used as a parameter of the theory, with no explicit relation with the specific microstructure. In this respect, compared to the model of semi-penetrable spheres introduced in Sec. II] Eq. (26) represents a semiphenomenological description of nanocomposite films. 

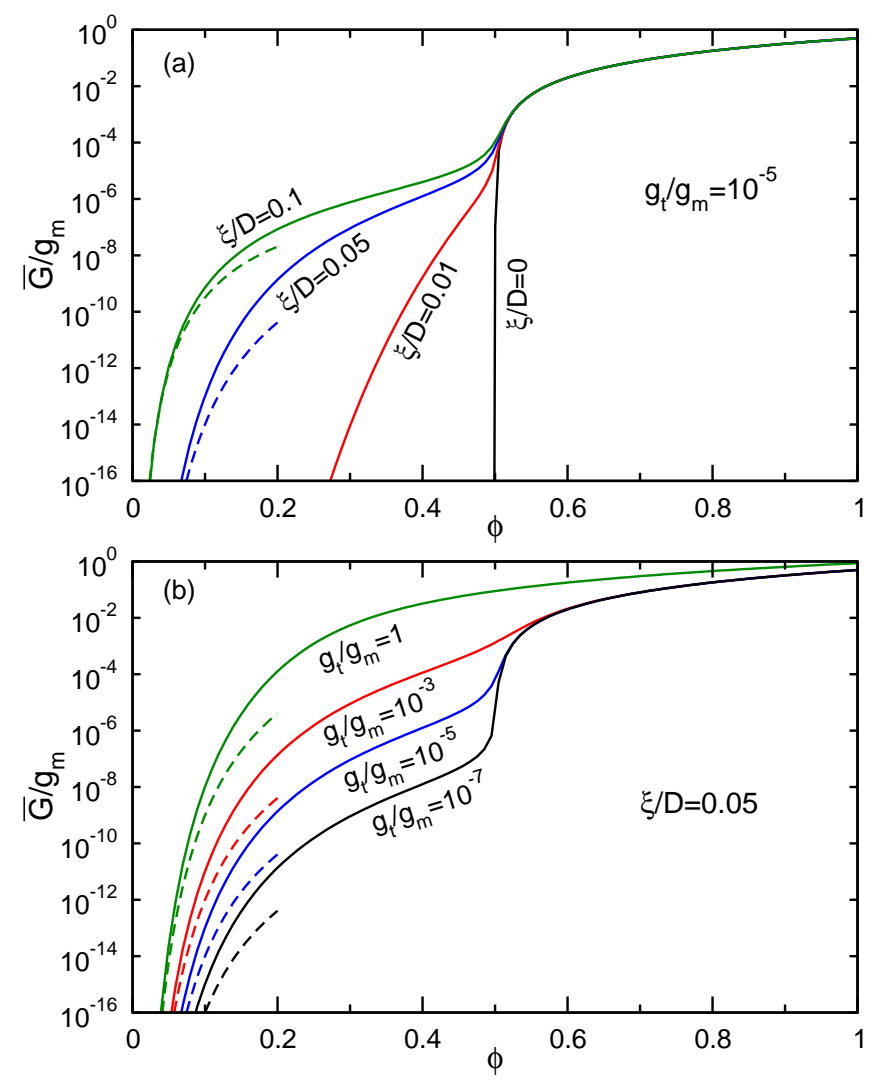

FIG. 2. (Color online) EMA conductance $\bar{G}$ as a function of the volume fraction $\phi$ of conducting spheres as obtained from numerical solutions of Eq. (26). The critical volume fraction $\phi_{c}$ for overlapping spheres is fixed at $\phi_{c}=0.5$. (a) $\bar{G}$ for different values of $\xi / D$ at fixed $g_{t} / g_{m}=10^{-5}$. For $\xi / D=0$ the EMA conductance follows the percolation behavior $\bar{G} \propto$ $\left(\phi-\phi_{c}\right)^{t}$, with $t=2$. Dashed lines are the low density $\bar{G}$ of Eq. (16). (b) $\bar{G}$ for different values of $g_{t} / g_{m}$ at fixed $\xi / D=$ 0.05 .

The $\phi$-dependence of $\bar{G}$, obtained by numerical solution of Eq. (26), is shown in Fig. 2 for different values of $\xi / D$ and $g_{t} / g_{m}$, with critical volume fraction fixed at $\phi_{c}=1 / 2$. For $\xi / D=0$, transport is purely percolative and the EMA conductance follows $\bar{G}=\left(g_{m} / \phi_{c}^{t}\right)\left(\phi-\phi_{c}\right)^{t}$ for $\phi \geq \phi_{c}$. In this region, the percolating behavior persists even for $\xi / D \neq 0$, while for $\phi<\phi_{c}$ the tunneling contributions become dominant and $\bar{G}$ asymptotically follows Eq. (16), as shown in Fig. 2(a). When plotted in a semi-logarithmic scale, the resulting $\phi$-dependence of $\bar{G}$ shows thus a characteristic double hump, commonly observed in granular metal films, which signals the metallic (percolating) and dielectric (tunneling) regimes. The double hump feature, and the conductance step at $\phi \simeq \phi_{c}$, depend however on the ratio $g_{t} / g_{m}$. For $g_{t} / g_{m}=1$, the EMA conductance decreases gradually as $\phi$ decreases without particular features at $\phi_{c}$, while a significant step becomes visible only for $g_{t} / g_{m} \ll 1$, as shown in Fig. 2(b).
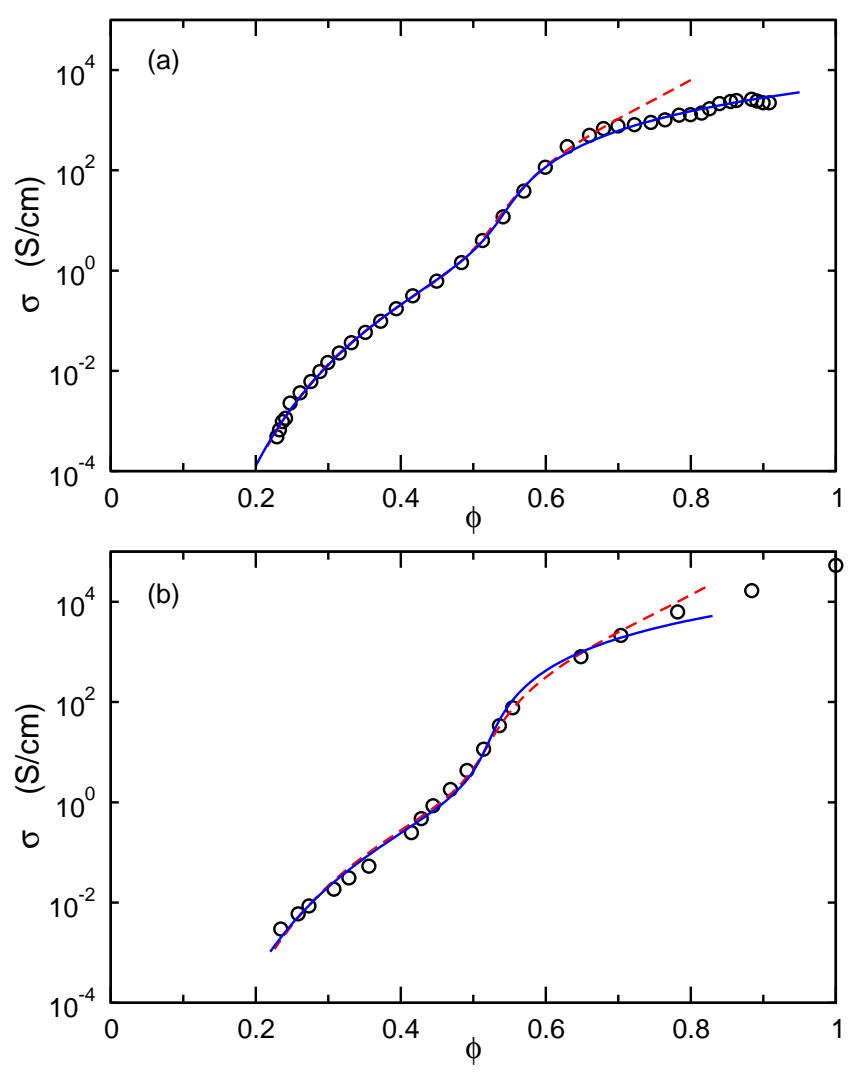

FIG. 3. (Color online) Measured conductivity $\sigma$ (open circles) as a function of $\mathrm{Ni}$ content for $\mathrm{Ni}^{-\mathrm{SiO}_{2}}$ granular films. Data are taken (a) from Ref. 14 and (b) from Ref. 1. Solid lines are fitting curves from solutions of Eq. (26). Dashed lines are least-square fit results of the cherry-pit EMA equation of Sec. IV Values of the fitting parameters are reported in Table III.

\section{A. Application to experiments}

To assess the relevance of our EMA model for real nanocomposite films, we solve Eq. (26) so to reproduce published data of the conductivity of several granular metal systems. To this end, we rewrite Eq. (26) in terms of the dimensionless conductance $g^{*}=\bar{G} / g_{m}$ which, besides $\phi$, depends on three parameters: $\phi_{c}, \xi / D$, and $g_{t} / g_{m}$. Since $g^{*}$ is independent of the system size, ${ }^{23,27}$ we define the EMA conductivity simply as $\bar{\sigma}=\Sigma g^{*}$, where $\Sigma$ is a fourth fitting parameter which has the dimension of a conductivity.

To find the values of $\phi_{c}, \xi / D, g_{t} / g_{m}$, and $\Sigma$ which best fit the experimental data, we apply a nonlinear leastsquares algorithm to the numerical solution of Eq. (26). Results of this procedure applied to $\mathrm{Ni}_{-} \mathrm{SiO}_{2}$ granular thick films are shown in Fig. 9] where the EMA conductivity (solid lines) is fitted to the room temperature conductivity data of $\mathrm{Ni}_{-} \mathrm{SiO}_{2}$ taken from Refs. 1 and 14 (open circles). The fitted percolation threshold for the case of Fig. 9(a), $\phi_{c} \simeq 0.52$, coincides with the value 

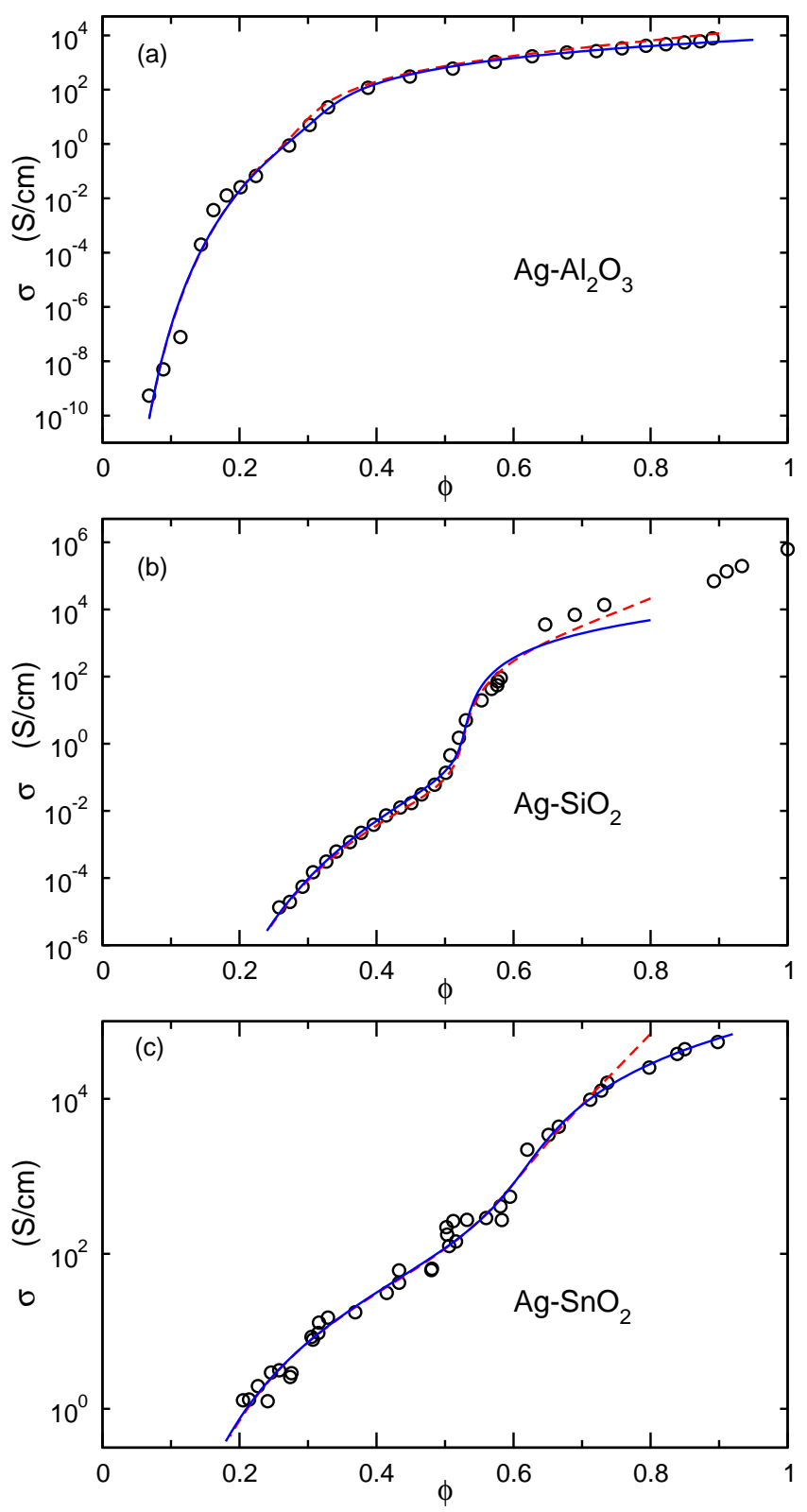

FIG. 4. (Color online) Measured conductivity $\sigma$ (open circles) as a function of $\mathrm{Ag}$ content for (a) $\mathrm{Ag}-\mathrm{Al}_{2} \mathrm{O}_{3} \frac{16}{\text { (b) }}$

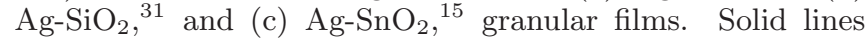
are fitting curves from solutions of Eq. (26). Dashed lines are least-squares fit results of the cherry-pit EMA equation of Sec. IV] Values of the fitting parameters are reported in Table

extracted in Ref. 14 from a fit with Eq. (1). This correspondence is not surprising because our EMA model has been constructed so as to reproduce the power-law behavior (1) with $t \simeq 2$ in the metallic regime. The value $\phi_{c} \simeq 0.51$ extracted from the data of Ref. 1 indicates that the percolation threshold is independent of the conditions of the co-sputtering deposition, while these seem to affect to some extent the conductivity above $\phi_{c}$.
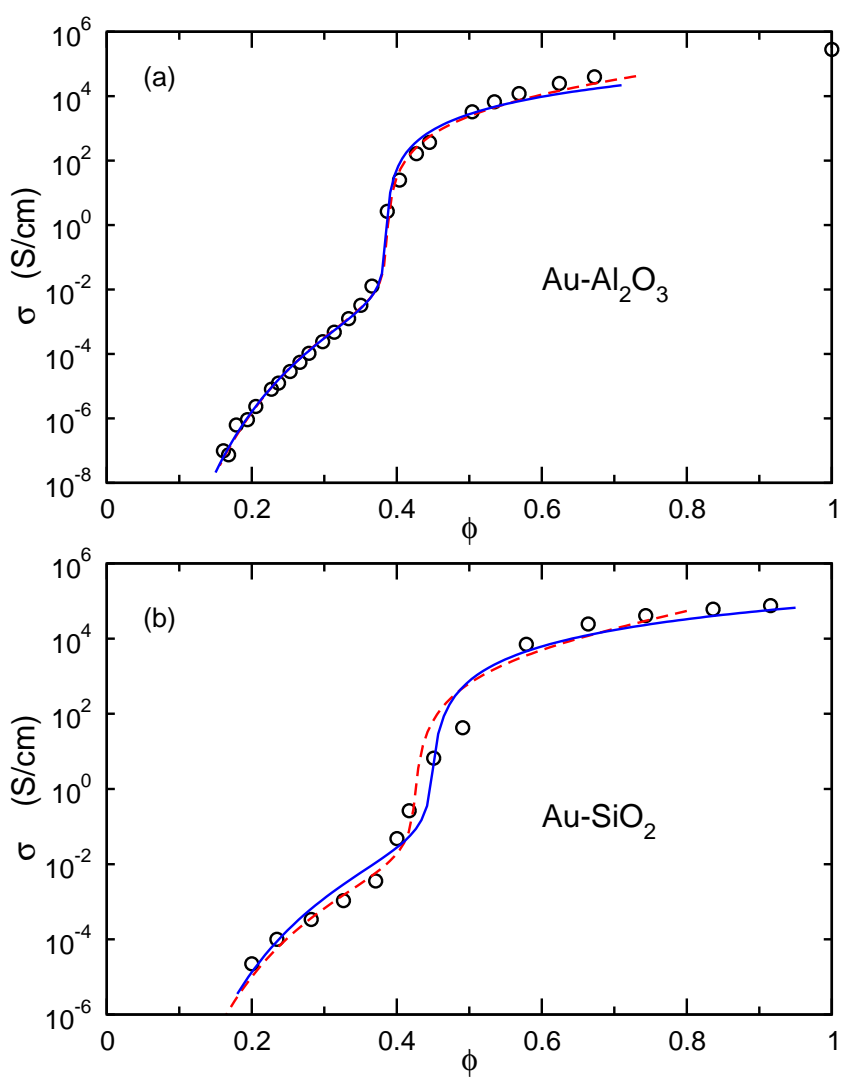

FIG. 5. (Color online) Measured conductivity $\sigma$ (open circles) as a function of $\mathrm{Au}$ content for (a) $\mathrm{Au}-\mathrm{Al}_{2} \mathrm{O}_{3} \stackrel{1}{\text { ․ }}$ and (b) $\mathrm{Au}-\mathrm{SiO}_{2}, \frac{31}{2}$ granular films. Solid lines are fitting curves from solutions of Eq. (26). Dashed lines are least-squares fit results of the cherry-pit EMA equation of Sec. IV] Values of the fitting parameters are reported in Table III

In the $\phi<\phi_{c}$ region, where tunneling dominates, the data from Ref. 14 are slightly better fitted than those from Ref. 1. For Ni-SiO 2 and other granular metals, the mean size of the metallic particles decreases as $\phi$ is smaller, $\frac{1}{-}$ while in our model we keep $D$ fixed. Hence, the better agreement of EMA for the case of Ref. 14 could be attributed to a lower rate of decrease of $D$ than for the film of Ref. 1. We note that the resulting $\xi / D \simeq 0.045$ and $\xi / D \simeq 0.052$ extracted respectively from Figs. 9(a) and 9(b) are nevertheless quite comparable, as also the tunneling to metal conductance ratio $g_{t} / g_{m}$ which is about $\sim 10^{-4}$ for both materials.

By following the same fitting procedure, we have reproduced the conductivity data of several nanogranular films composed of noble metal1,15,16,31 or magnetic fillers $12,32-34$ with different dielectric matrices, as shown in Figs. 10,13. The corresponding fitting parameters are reported in Table III. Despite the simplicity of Eq. (26), the overall quality of the fits is remarkable. In particular, EMA captures well the dielectric regime below $\phi_{c}$ and the transition to the metallic regime in the vicinity of $\phi_{c}$. Some deviations from the experimental data 

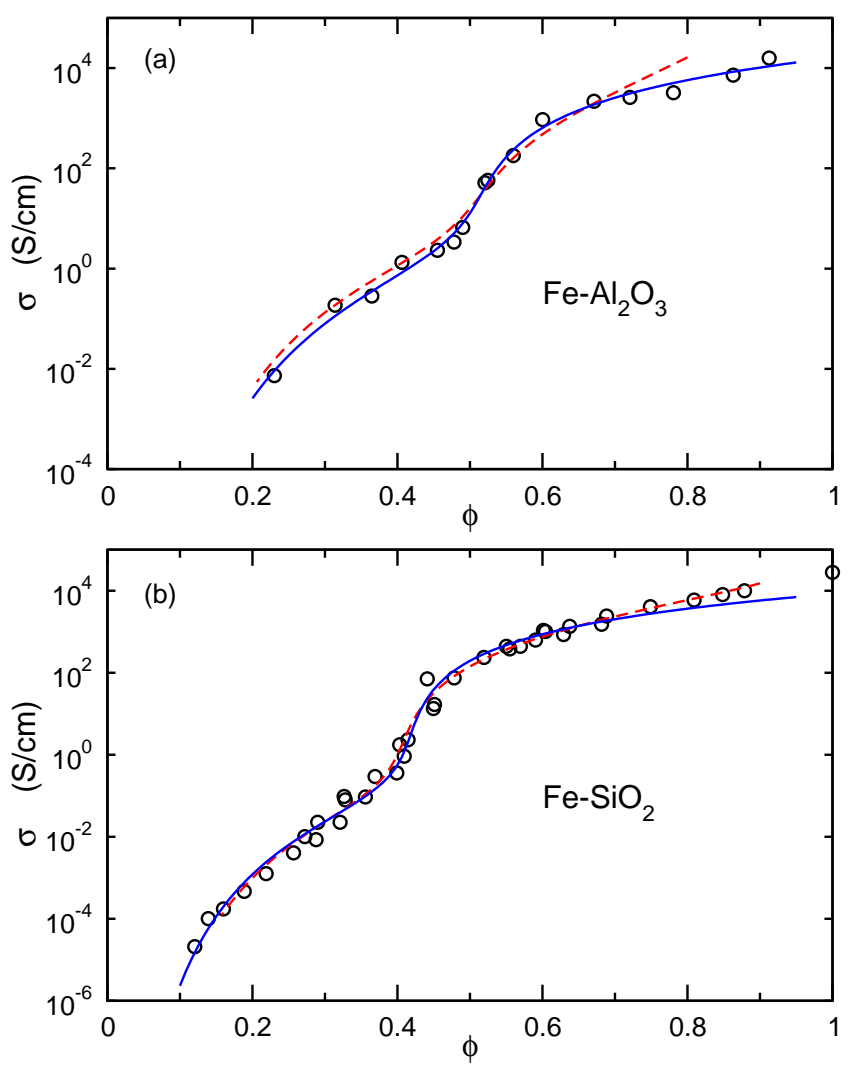

FIG. 6. (Color online) Measured conductivity $\sigma$ (open circles) as a function of $\mathrm{Fe}$ content for (a) $\mathrm{Fe}-\mathrm{Al}_{2} \mathrm{O}_{3}, \frac{32}{2}$ and (b) $\mathrm{Fe}-\mathrm{SiO}_{2},{ }^{12}$ granular films. Solid lines are fitting curves from solutions of Eq. (26). Dashed lines are least-squares fit results of the cherry-pit EMA equation of Sec. IV Values of the fitting parameters are reported in Table III

are visible in the large $\phi$ region, especially for $\mathrm{Ag}-\mathrm{SiO}_{2}$ in Fig. 10(b), due to the imposed power-law behavior (11) which is expected to be valid only for $\phi$ immediately above $\phi_{c}$. Concerning the transition region, we point out that all films here considered have thicknesses in the micrometer range, justifying thus the use of the transport exponent value $t=2$ valid for three dimensional percolating systems. By using the EMA value $t=1$ for the transport exponent, we obtain that the overall quality of the fits does not change appreciably: only in the transition region about $\phi_{c}$ the fitting curves have occasionally a more abrupt variation, but the fitted values of $\phi_{c}$ and $\xi / D$ do not show appreciable variations $\frac{35}{3}$

From the values of $\phi_{c}$ reported in Table III we see that the percolation threshold ranges between 0.3 and 0.62 , with no correlation with the type of insulating phase. This result confirms earlier observations that the critical volume fraction depends on the particular combination of metal and insulator constituting the film.$\underline{1}$ Similarly, also the conductivity step at about $\phi_{c}$, parametrized by $g_{t} / g_{m}$, does not show any particular trend. In this respect, we note that depending on the specific composite
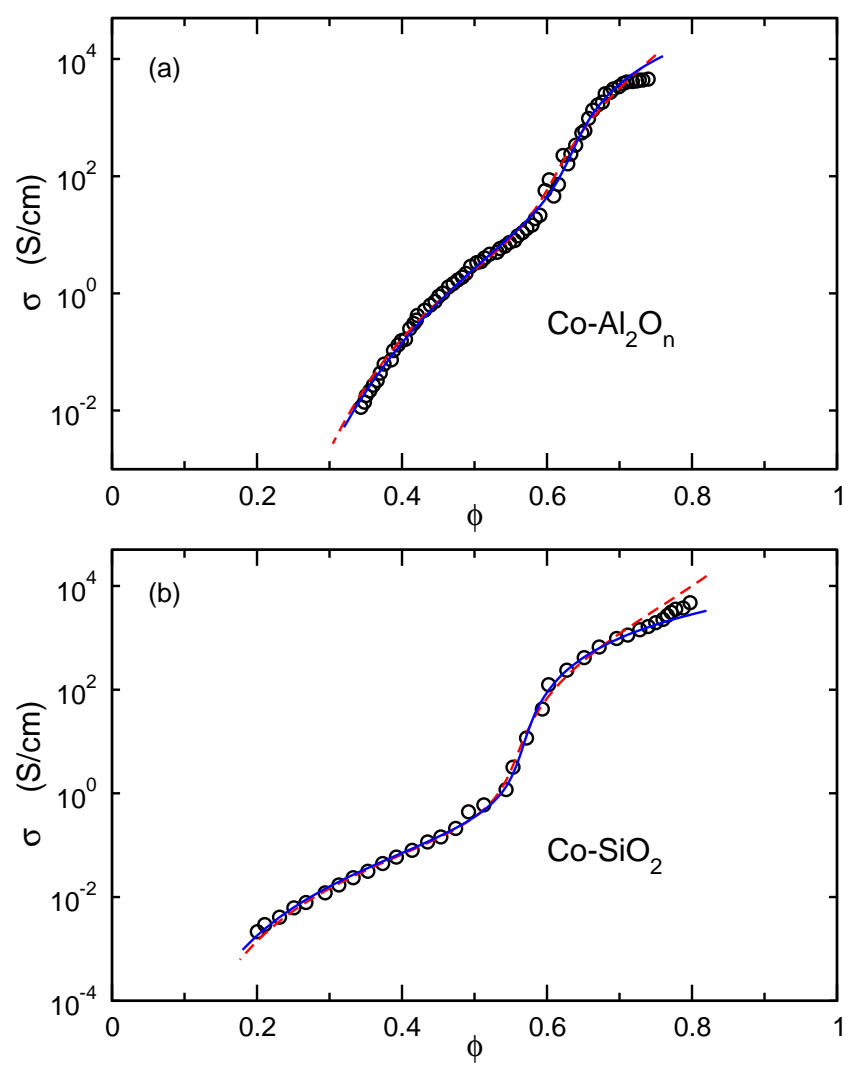

FIG. 7. (Color online) Measured conductivity $\sigma$ (open circles) as a function of Co content for (a) $\mathrm{Co}-\mathrm{Al}_{2} \mathrm{O}_{n}, \frac{33}{3}$ and (b) $\mathrm{Co}-\mathrm{SiO}_{2}, \stackrel{34}{,}$ granular films. Solid lines are fitting curves from solutions of Eq. (26). Dashed lines are least-squares fit results of the cherry-pit EMA equation of Sec. IV] Values of the fitting parameters are reported in Table III

$g_{t} / g_{m}$ ranges between $\sim 10^{-3}$ and $\sim 10^{-7}$. As mentioned previously, we expect $g_{t}$ to be smaller than $g_{m}$ due to particle interfacial barrier and particle charging and Coulomb interaction effects. However, further reduction of $g_{t}$ can be induced also by non-random distributions of metal particle separations, as reported for example in Ref. 36 where gaps of the order of one nanometer in the interparticle spacing have been observed. For a nonzero gap $\Delta$, indeed, we can replace the lowest limit of integration in the second term of Eq. (11) by $D_{\Delta}=D+\Delta \cdot 37$ Thus, if we rewrite the tunneling conductance in (4) as

$$
g_{t} \exp \left[-\frac{2(r-D)}{\xi}\right]=g_{t}^{*} \exp \left[-\frac{2\left(r-D_{\Delta}\right)}{\xi}\right],
$$

where $g_{t}^{*}=\exp (-2 \Delta / \xi)$ is a rescaled prefactor, the function $I$ of Eq. (15) becomes:

$$
I^{*}=8 \phi\left(\frac{D_{\Delta}}{D}\right)^{3}\left\{\left[1+\frac{\xi}{2 D_{\Delta}} \ln \left(\frac{g_{t}^{*}+\bar{G}}{\bar{G}}\right)\right]^{3}-1\right\},
$$

which in the dilute limit gives the same asymptotic $\bar{G}$ of Eq. (16) with $g_{t}$ replaced by $g_{t}^{*}$. If we interpret the 
values of $g_{t} / g_{m}$ reported in Table 【II as actually representing $g_{t}^{*} / g_{m}$, we can easily explain values as small as $\sim 10^{-7}$, as observed for example for composites with Au. Indeed, even assuming that $g_{t} \sim g_{m}$, from $g_{t}^{*} / g_{m} \sim$ $\exp (-2 \Delta / \xi) \sim 10^{-7}$ we get $\Delta \sim 0.8 \mathrm{~nm}$ for tunneling decay lengths of about $0.1 \mathrm{~nm}$.

Turning to the dielectric regime of $\sigma$ identified by the hump at $\phi<\phi_{c}$ in Figs. 9, [13, we note that the tunneling decay length for a rectangular barrier is $\xi=\hbar / \sqrt{2 m \varphi}$, where $m$ is the electron mass and $\varphi$ is the tunnel barrier height. We estimate $\varphi$ as the difference between the work function of the metal and the electron affinity of the dielectric. Since the work function for the metals considered here ranges from about $4.5 \mathrm{eV}(\mathrm{Fe})$ to about $5.4 \mathrm{eV}(\mathrm{Au}) \stackrel{38}{\underline{36}}$ while the electron affinities for $\mathrm{Al}_{2} \mathrm{O}_{3}$ and $\mathrm{SiO}_{2}$ are respectively $\sim 1.35 \mathrm{eV}$ and $\sim 1 \mathrm{eV}, \frac{39}{1}$ we obtain that the tunneling decay length is $\xi \simeq 0.1 \mathrm{~nm}$ for the composites with $\mathrm{Al}_{2} \mathrm{O}_{3}$ and $\mathrm{SiO}_{2}$. As the fitted values of $\xi / D$ range from 0.03 to about 0.1 (see Table III), we infer that for this class of composites the mean size of metal particles is comprised between $D \sim 1 \mathrm{~nm}$ and $D \sim 3 \mathrm{~nm}$. This estimate is in fair accord with the observed particle sizes in these systems, as shown in Table II where we compare our results of $D$ with measured values of the mean particle sizes.

For the $\mathrm{Ag}-\mathrm{SnO}_{2}$ system, the large electron affinity of the oxide semiconductor $\mathrm{SnO}_{2}$ (about $4.3-4.5 \mathrm{eV} \stackrel{40}{\underline{4}}$ ) together with the work function $\sim 4.6 \mathrm{eV}$ for $\mathrm{Ag} \stackrel{38}{=}$ gives $\xi \simeq 0.4-0.7 \mathrm{~nm}$. From $\xi / D=0.09$ we obtain thus $D \simeq 4.4-7.8 \mathrm{~nm}$, which is comparable with $D \simeq 3-7 \mathrm{~nm}$ measured in samples with $\phi<0.42 .15$ We note that using the point particle limit of Eq. (10) (with $a_{3} \simeq 1.41$ ) to find $\xi / D$ from the conductivity data leads to the slightly larger estimate $\xi / D \simeq 0.13: \underline{15}$

\section{EMA CHERRY-PIT MODEL}

The EMA model discussed in Sec. III treats the transition between the metallic and dielectric regimes in a phenomenological way by introducing a critical volume fraction whose value is found by fitting the experiments. In the model illustrated in Fig. 1 we have however assumed that the metallic particles are allowed to overlap to some extent, and that the degree of overlapping determines the value of $\phi_{c}$. Furthermore, in deriving Eq. (26) we have considered the metallic particles as completely uncorrelated by setting $g_{2}(r)=1$ for all particle contents lower than $\phi_{c}$. To include explicitly particle overlaps and local correlation, we consider a cherry-pit model in which each metallic sphere of diameter $D$ is composed by an impenetrable core of diameter $\lambda D$ surrounded by a penetrable concentric shell of thickness $(1-\lambda) D / 2 \stackrel{29}{2}$ Any two given metallic spheres can thus overlap as long as their respective hard cores do not. The parameter $\lambda$ ranges between 0 and 1 , which defines the limits of fully penetrable and totally impenetrable spheres, respectively. For equilibrium distributions of cherry-pit spheres, the criti- cal volume fraction for percolation of overlapping spheres varies thus between $\phi_{c} \simeq 0.29$ for $\lambda=0$ and $\phi_{c} \simeq 0.64$ for $\lambda=1, \underline{29}$ (see also Fig. 8) consistently with the range of $\phi_{c}$ values we have obtained in Sec. IIIA.

To apply the general EMA equation (11) to the case of equilibrium cherry-pit spheres, we note that the radial distribution function $g_{2}(r)$ is that of hard-core spheres of diameter $\lambda D, g_{2}^{\mathrm{hc}}(r ; \lambda D)$, as the penetrable shell has no effects on the equilibrium distribution. Furthermore, to relate the fractional coverage $\phi$ of the cherry-pit spheres with the number density $\rho$, we use the approximate but accurate formula: 29,41

$$
\phi=1-\left(1-\eta \lambda^{3}\right) \exp \left[-\frac{\left(1-\lambda^{3}\right) \eta}{\left(1-\eta \lambda^{3}\right)^{3}}\right] A(\eta, \lambda)
$$

with

$$
\begin{aligned}
A(\eta, \lambda)= & \exp \left\{-\frac{\eta^{2} \lambda^{3}(\lambda-1)}{2\left(1-\eta \lambda^{3}\right)^{3}}\left[\left(7 \lambda^{2}+7 \lambda-2\right)\right.\right. \\
& \left.\left.-2 \eta \lambda^{3}\left(7 \lambda^{2}-5 \lambda+1\right)+\eta^{2} \lambda^{6}\left(5 \lambda^{2}-7 \lambda+2\right)\right]\right\},
\end{aligned}
$$

where we have introduced the dimensionless density $\eta=$ $\pi D^{3} \rho / 6 \underline{\underline{42}}$ Equation (11) reduces in this way to:

$$
\frac{Z(\phi, D) g_{m}^{1 / t}}{\bar{G}^{1 / t}+g_{m}^{1 / t}}+\frac{24 \eta}{D^{3}} \int_{D}^{\infty} d r r^{2} \frac{g_{2}^{\mathrm{hc}}(r ; \lambda D)}{\left(\bar{G} / g_{t}\right) e^{\frac{2(r-D)}{\xi}}+1}=2,
$$

where we have corrected the first term by using the transport exponent $t$, as done in Eq. (26). Since $g_{2}^{\text {hc }}(r ; \lambda D)=$ 0 for $r<\lambda D$, the coordination number function for overlapping spheres in Eq. (24) is an integral between $\lambda D$ and $D$ :

$$
Z(\phi, D)=\frac{24 \eta}{D^{3}} \int_{\lambda D}^{D} d r r^{2} g_{2}^{\mathrm{hc}}(r ; \lambda D)
$$

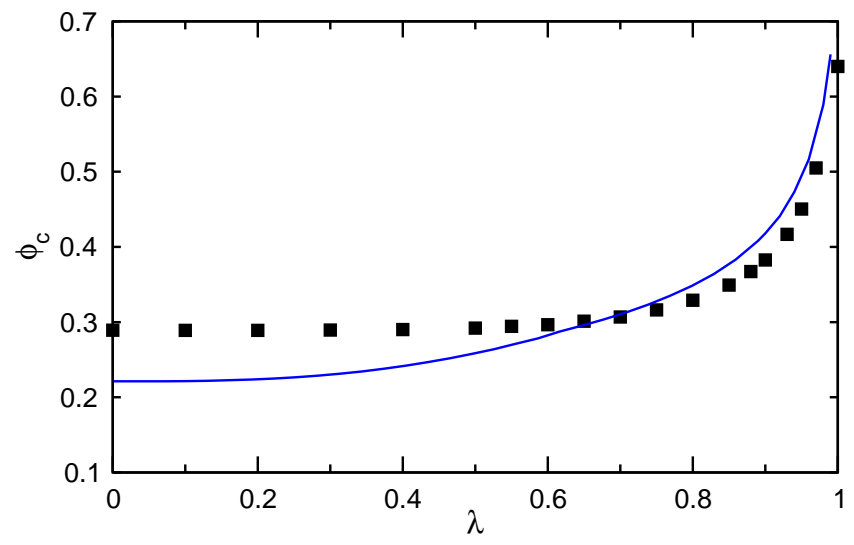

FIG. 8. (Color online) Critical volume fraction $\phi_{c}$ as a function of the impenetrability parameter $\lambda$ of the cherry-pit model. Solid line is the EMA $\phi_{c}$ obtained from $Z\left(\phi_{c}, D\right)=2$. Filled squares are Monte Carlo results of Ref. 44 in which Eqs. (22) and (23) are used to relate $\phi_{c}$ with the critical density $\eta_{c}$. 
TABLE I. Values of $\phi_{c}, \xi / D, g_{t} / g_{m}$, and $\Sigma$ that best fit the measured conductivity data of Refs. 1, 12, 14 16, 31-34 obtained using the EMA semi-phenomenological model of Sec. III (model A) and the EMA cherry-pit model of Sec. IVV (model B). The values in parentheses are the fitted values of the impenetrability parameter $\lambda$ of the EMA cherry-pit model.

\begin{tabular}{|c|c|c|c|c|c|c|c|c|}
\hline \multirow[t]{2}{*}{ Material } & \multicolumn{2}{|r|}{$\phi_{c}$} & \multicolumn{2}{|c|}{$\xi / D$} & \multicolumn{2}{|c|}{$g_{t} / g_{m}$} & \multicolumn{2}{|c|}{$\Sigma(\mathrm{S} / \mathrm{cm})$} \\
\hline & model A & model B & model A & model B & model A & model B & model A & model B \\
\hline $\mathrm{Ni}^{-\mathrm{SiO}_{2}}$ (Ref. 14) & 0.52 & $0.49(0.957)$ & 0.045 & 0.046 & $2.9110^{-4}$ & $3.4310^{-3}$ & $5.4810^{3}$ & $2.7810^{2}$ \\
\hline $\mathrm{Ni}^{-\mathrm{SiO}_{2}}$ (Ref. 1) & 0.51 & $0.486(0.954)$ & 0.052 & 0.051 & $7.8410^{-5}$ & $1.4910^{-3}$ & $1.3110^{4}$ & $6.1810^{2}$ \\
\hline $\mathrm{Ag}-\mathrm{Al}_{2} \mathrm{O}_{3}$ (Ref. 16) & 0.3 & $0.27(0.58)$ & 0.046 & 0.047 & $2.910^{-3}$ & $2.6910^{-3}$ & $1.4610^{3}$ & $6.9410^{2}$ \\
\hline $\mathrm{Ag}-\mathrm{SiO}_{2}$ (Ref. 31) & 0.52 & $0.50(0.96)$ & 0.03 & 0.032 & $6.2610^{-6}$ & $4.7310^{-5}$ & $1.7510^{4}$ & $9.1410^{2}$ \\
\hline $\mathrm{Ag}-\mathrm{SnO}_{2}$ (Ref. 15) & 0.59 & $0.53(0.97)$ & 0.091 & 0.094 & $4.6010^{-4}$ & $2.0310^{-2}$ & $2.1210^{5}$ & $2.9110^{3}$ \\
\hline $\mathrm{Au}-\mathrm{Al}_{2} \mathrm{O}_{3}$ (Ref. 1) & 0.38 & $0.38(0.862)$ & 0.043 & 0.045 & $1.3510^{-7}$ & $6.1110^{-7}$ & $3.0210^{4}$ & $7.2310^{3}$ \\
\hline $\mathrm{Au}-\mathrm{SiO}_{2}$ (Ref. 31) & 0.45 & $0.42(0.90)$ & 0.048 & 0.056 & $7.7410^{-7}$ & $2.4510^{-6}$ & $5.1410^{4}$ & $3.9710^{3}$ \\
\hline $\mathrm{Fe}-\mathrm{Al}_{2} \mathrm{O}_{3}$ (Ref. 32) & 0.50 & $0.48(0.95)$ & 0.061 & 0.064 & $1.2910^{-4}$ & $3.1410^{-3}$ & $1.6010^{4}$ & $7.7410^{2}$ \\
\hline $\mathrm{Fe} \mathrm{SiO}_{2}$ (Ref. 12) & 0.41 & $0.39(0.878)$ & 0.078 & 0.078 & $2.8910^{-5}$ & $2.0410^{-4}$ & $4.0310^{3}$ & $5.1910^{2}$ \\
\hline $\mathrm{Co}-\mathrm{Al}_{2} \mathrm{O}_{n}$ (Ref. 33 ) & 0.62 & $0.56(0.98)$ & 0.026 & 0.026 & $1.2210^{-4}$ & $3.9610^{-3}$ & $2.1810^{5}$ & $210^{5}$ \\
\hline Co-SiO ${ }_{2}$ (Ref. 34) & 0.56 & $0.53(0.97)$ & 0.097 & 0.095 & $1.0210^{-5}$ & $2.710^{-4}$ & $1.5010^{4}$ & $4.2210^{2}$ \\
\hline
\end{tabular}

TABLE II. Estimated particle sizes $D$ extracted from the $\xi / D$ values of Table III using $\xi=0.4-0.7 \mathrm{~nm}$ for $\mathrm{Ag}_{-} \mathrm{SnO}_{2}$ and $\xi=0.1 \mathrm{~nm}$ for all other cases (see main text). Unless otherwise indicated, the experimentally determined values of $D$ represent the mean particle sizes extracted from scanning or transmission electron microscopy in the dielectric regime (i.e., below the percolation threshold). Missing entries mean that the corresponding publications do not report measurements of particle sizes for the granular film considered.

\begin{tabular}{|c|c|c|}
\hline \multirow[t]{2}{*}{ Material } & \multicolumn{2}{|r|}{$D(\mathrm{~nm})$} \\
\hline & Theory & Experiments \\
\hline $\mathrm{Ni}-\mathrm{SiO}_{2}$ (Ref. 14) & 2.2 & \\
\hline $\mathrm{Ni}^{-\mathrm{SiO}_{2}}$ (Ref. 1) & 2 & $2.5-4($ for $0.3 \leq \phi \leq 0.55)$ \\
\hline $\mathrm{Ag}-\mathrm{Al}_{2} \mathrm{O}_{3}$ (Ref. 16) & 2.2 & \\
\hline $\mathrm{Ag}-\mathrm{SiO}_{2}$ (Ref. 31) & 3.3 & $\sim 10(\text { at } \phi=0.38)^{\mathrm{a}}$ \\
\hline $\mathrm{Ag}-\mathrm{SnO}_{2}$ (Ref. 15) & $4.4-7.8$ & $3-7($ for $\phi<0.42)$ \\
\hline $\mathrm{Au}-\mathrm{Al}_{2} \mathrm{O}_{3}$ (Ref. 1 ) & 2.3 & $1.8-3.5($ for $0.15 \leq \phi \leq 0.35)$ \\
\hline $\mathrm{Au}-\mathrm{SiO}_{2}$ (Ref. 31) & 2 & $2-8(?)($ for $0.1 \leq \phi \leq 0.4)$ \\
\hline $\mathrm{Fe}-\mathrm{Al}_{2} \mathrm{O}_{3}$ (Ref. 32) & 1.7 & $1-3(\text { at } \phi=0.45)^{\mathrm{b}}$ \\
\hline $\mathrm{Fe}-\mathrm{SiO}_{2}$ (Ref. 12) & 1.3 & $1-2.5($ for $\phi \leq 0.3)$ \\
\hline $\mathrm{Co}-\mathrm{Al}_{2} \mathrm{O}_{n}$ (Ref. 33 ) & 3.8 & \\
\hline Co-SiOH $($ Ref. 34) & 1 & $3.5-4.8(\text { for } 0.2 \leq \phi \leq 0.35)^{\mathrm{b}}$ \\
\hline
\end{tabular}

a Our estimate from Fig. 2(b) of Ref. 31 .

b From fits of magnetization data.

From the above equation we can already determine how the percolation threshold $\phi_{c}$ depends on the impenetrability parameter $\lambda$ by using the EMA relation $Z\left(\phi_{c}, D\right)=$ 2 derived in Sec. IIB. To this end, we use in Eq. (25) $g_{2}^{\text {hs }}(r ; \lambda D)$ as given by the accurate expression for the radial distribution function of hard spheres derived in Ref. 43, and apply Eqs. (22) and (23) to find $\phi_{c}$ from the critical density $\eta_{c}$. The resulting critical volume fraction compares relatively well with the numerical calculations for $\lambda \gtrsim 0.5$, as seen in Fig. 8 where the filled squares are the Monte Carlo results of Ref. 44. By comparing the values of $\phi_{c}$ reported in Fig. 8 with those listed in Table III. from which we see that $\phi_{c}>0.4$ with the exception of $\mathrm{Ag}-\mathrm{Al}_{2} \mathrm{O}_{3}$, we infer that the percolation thresholds of nanogranular films are reproduced by the EMA cherrypit model with $\lambda \gtrsim 0.85$, which means that the spheres have generally little overlap. We obtain even (slightly) smaller overlaps if we compare the experimental percolation thresholds with the Monte Carlo $\phi_{c}$ of Fig. 8.

Although we do not expect that the detailed morphology of real granular metal films is fully reproduced by equilibrium dispersions of cherry-pit spheres, these seem nevertheless to capture some critical aspects of the microstructure and its evolution with $\phi$. We note also that other simple microscopic descriptions, as for example the equilibrium permeable spheres model for which expressions of $g_{2}(r)$ and of the volume fraction exist, $\stackrel{45}{w}$ may equally be used though they are of less practical implementation.

We proceed to apply the EMA cherry-pit model to the experimental data of Figs. 9.13 by using $\xi / D, g_{t} / g_{m}, \Sigma$, and $\lambda$ as fitting parameters and $t=2$ fixed. We first invert numerically Eqs. (22) and (23) to extract $\eta$ from the measured $\phi$ values, and subsequently we solve iteratively Eqs. (24) and (25) by using the model $g_{2}^{\mathrm{hs}}(r ; \lambda D)$ of Ref. 43. The results of nonlinear least-squares fits are shown by dashed lines in Figs. 9, 13, and the values of the fitting parameters that best reproduce the measured $\sigma$ are reported in Table III. We see that the cherry-pit model confirms the results obtained in the previous section. In particular, the two fitting curves (solid and dashed lines) are practically indistinguishable in both the dielectric regime and the transition region about $\phi_{c}$, and very similar values of $\xi / D$ and $\phi_{c}$ are obtained from the two methods, as shown in Table [II] Furthermore, the quantitative accord with the experimental 
data for $\phi \lesssim \phi_{c}$ confirms our assumption that local particle correlations are marginal in the dielectric regime. For metallic contents well above $\phi_{c}$, the conductivity of cherry-pit model is systematically larger than that of the semi-phenomenological approach of Sec. III and gives occasionally better fits, as seen in Figs. 9(b), 10(b), and 12(b). We note however that the radial distribution function of Ref. 43 becomes inaccurate for packing fractions $\lambda^{3} \eta$ of the hard cores larger than $\sim 0.5$. Assuming for example $\lambda=0.95$, as is the case of $\mathrm{Fe}-\mathrm{Al}_{2} \mathrm{O}_{3}$, in terms of the fractional coverage this limitation translates into questionable results for $\phi \gtrsim 0.6$.

\section{DISCUSSION AND CONCLUSIONS}

The central result of this paper is that the two fundamentally different transport regimes of percolation and tunneling, which are simultaneously observed in many conducting nanocomposite films, find a natural explanation within a single theoretical framework. We have shown that quite general considerations on the nature of the interparticle electrical connectedness and on the distribution of the metallic phase are sufficient to describe quantitatively the dc conductivity $\sigma$ of several granular metal films. In particular, the semi-phenomenological EMA equation derived in Sec. III represents a simple, yet efficient, tool to analyze the $\phi$-dependence of $\sigma$ and to estimate the values of the microscopic parameters that govern the observed conductivity behaviors.

In formulating the minimal model of Sec. II] we have made different assumptions with the intent of keeping the theory as essential as possible. One of such assumptions concerns the direct tunneling decay of Eq. (4) in which we neglect particle charging and Coulomb interaction effects. As discussed in Sec II, these become important as the temperature is lowered below room temperature and/or as the particle size decreases 10 For particle sizes of the order of a few nanometers, and well below the percolation threshold, Coulomb effects become relevant also at room temperature, so that they could modify to some extent the $\xi / D$ values reported in Table III. A generalization of the present EMA approach as to include Coulomb gap effects would permit us to study on equal footing both the concentration and the temperature dependencies of transport, while these two are generally treated separately. In this respect, measured $\sigma$ dependencies on both concentration and temperature, as those reported for example in Refs. 1, 14, 15, and 34, would find a more complete, and unified, theoretical understanding.

In Sec. II we have also assumed that the metallic particles are spherical and of fixed diameter. Although we do not expect that small deviations from sphericity would have any important effect, metallic inclusions with high aspect-ratios can change appreciably the location of $\phi_{c}$ and the low-density tunneling regime. For example, the tunneling conductivity of dispersions of rod particles of diameter $D$ and length $L \gg D$ scales approximately as $\sigma \propto \exp \left(-D^{2} / \xi \phi L\right)$ for isotropic orientation of rods 20 The effect of elongated particles, as those observed in some granular films with magnetic particles, can nevertheless be investigated by applying EMA to high aspectratio fillers, as done for the tunneling case in Refs. 26 and 46

Concerning the assumption of fixed particle size, we note that some composite films show a more or less pronounced reduction of the mean particle size $D$ as $\phi$ decreases, as reported for example in Ref. 1 and in Table II. This effect can be included in Eq. (11) by considering an explicit $\phi$-dependence of $D$ which simulates the observed one. In principle, it is possible to consider within EMA also the effect of particle size polydispersity, although this would require detailed knowledge of the size distribution and its possible dependence on $\phi \stackrel{47}{=}$ In the absence of these informations, the theoretical estimates of Table II can be tentatively interpreted in terms of effective sizes $D_{\text {eff }}$ of polydisperse particles. It is not difficult to estimate $D_{\text {eff }}$ from the solution of Eq. (14) for asymptotically small $\bar{G}$, which is given by the last line of Eq. (16) with $D$ replaced by $D_{\text {eff }}=\sqrt[3]{\left\langle D^{3}\right\rangle}$. Particle size distributions with long tails for large $D$ may thus have $D_{\text {eff }}$ considerably larger than the mean $\langle D\rangle$. We note however that to coherently describe the effect of particle size polydispersity, charging and Coulomb interactions should be considered as well, since these become increasingly important as particle sizes are smaller.

Finally, we point out that although the cherry-pit model of Sec. IV] includes local correlations induced by the particle hard-cores, it ignores possible long-range correlations and is not suitable to describe particle clustering or aggregation effects. Although the granular films here considered do not appear to show long-range correlations, the general two-site EMA equation (11) allows us to include at least partially these effects through suitable choices of the radial distribution function $g_{2}(r)$. Aggregation induced by effective particle attractions can be modeled for example by attractive square-well potentials, for which approximate expressions for $g_{2}(r)$ are available $\underline{48}$ In the case of tunneling, the resulting EMA conductance is in excellent overall agreement with numerical simulations for a wide range of potential profiles, as shown in Ref. 25. Another possible route to simulate phenomenologically particle aggregation and clustering is to consider simple square-well models of $g_{2}(r) \stackrel{25,46}{\longleftarrow}$ whose values for $r$ lower and larger than some characteristic correlation distance can be adjusted to fit the measured conductivity.

We conclude by mentioning that even though we have formulated the theory to describe granular thick films as three dimensional systems, it is in principle not difficult to modify EMA to (quasi) two-dimensional systems, so to describe transport in thin granular films as those studied for example in Refs. 17 and 18 . 


\section{ACKNOWLEDGMENTS}

The author thanks I. Balberg, A. P. Chatterjee, and M. C. Salvadori for useful comments and Z.-Q. Li for kindly providing the original conductivity data of Ref. 15 .

1 B. Abeles, P. Sheng, M. D. Coutts, and Y. Arie, Adv. Phys. 24, 407 (1975).

2 G. A. Niklasson and C. G. Granqvist, J. Appl. Phys. 55, 3382 (1984).

3 X. Batlle and A. Labarta, J. Phys. D: Appl. Phys. 35, R15 (2002).

4 D. Stauffer and A. Aharony, Introduction to Percolation Theory (Taylor and Francis, London, 1994).

${ }^{5}$ M. Sahimi, Heterogeneous Materials I. Linear Transport and Optical Properties (Springer, New York, 2003).

6 A. G. Hunt, Percolation Theory for Flow in Porous Media (Springer, Berlin, 2005).

7 C. H. Seager and G. E. Pike, Phys. Rev. B 10, 1435 (1974).

8 A. L. Efros and B. I. Shklovskii, J. Phys. C 8, L49 (1975).

9 B. I. Shklovskii and A. L. Efros, Electronic Properties of Doped Semiconductors (Springer-Verlag, New York, 1988).

10 I. S. Beloborodov, A. V. Lopatin, V. M. Vinokur, and K. B. Efetov, Rev. Mod. Phys. 79, 469 (2007).

11 M. Pollak, M. Ortuño, and A. Frydman, The Electron Glass (Cambridge University Press, New York, 2013)

12 S. Honda, T. Okada, M. Nawate, and M. Tokumoto, Phys. Rev. B 56, 14566 (1997).

13 S. P. McAlister, A. D. Inglis, and P. M. Kayll, Phys. Rev. B 31, 5113 (1985).

14 D. Toker, D. Azulay, N. Shimoni, I. Balberg, and O. Millo, Phys. Rev. B 68, 041403(R) (2003); I. Balberg, D. Azulay, D. Toker, and O. Millo, Int. J. Modern Phys. B 18, 2091 (2004).

15 Y.-F. Wei and Z.-Q. Li, Appl. Phys. Lett. 102, 131911 (2013).

16 I. Balberg, D. Azulay, Y. Goldstein, J. Jedrzejewski, G. Ravid, and E. Savir, Eur. Phys. J. B 86, 428 (2013).

17 M. C. Salvadori, M. Cattani, F. S. Teixeira, and I. G. Brown, Appl. Phys. Lett. 93, 073102 (2008); F. S. Teixeira, M. C. Salvadori, M. Cattani, and I. G. Brown, J. Vac. Sci. Technol. A 28, 818 (2010); M. C. Salvadori, F. S. Teixeira, L. G. Sgubin, M. Cattani, and I. G. Brown, Nucl. Instrum. Meth. B 310, 32 (2013).

18 S. Fostner, R. Brown, J. Carr, and S. A. Brown, Phys. Rev. B 89, 075402 (2014).

19 I. Balberg, Phys. Rev. Lett. 59, 1305 (1987).

20 G. Ambrosetti, C. Grimaldi, I. Balberg, T. Maeder, A. Danani, and P. Ryser, Phys. Rev. B 81, 155434 (2010).

${ }^{21}$ G. Ambrosetti, I. Balberg, and C. Grimaldi, Phys. Rev. B 82, 134201 (2010).

22 J. Inoue and S. Maekawa, Phys. Rev. B 53, R11927 (1996).

23 C. Grimaldi, Europhys. Lett. 96, 36004 (2011).

24 B. Nigro, C. Grimaldi, and P. Ryser, Phys. Rev. E 85, 011137 (2012).

25 B. Nigro, C. Grimaldi, M. A. Miller, P. Ryser, and T. Schilling, J. Chem. Phys. 136, 164903 (2012).
26 B. Nigro, C. Grimaldi, M. A. Miller, P. Ryser, and T. Schilling, Phys. Rev. E 88, 042140 (2013).

27 F. Y. Wu, J. Phys. A: Math. Gen. 37, 6653 (2004).

28 J.-P. Hansen and I. R. McDonald, Theory of Simple Liquids (Elsevier, New York, 2006).

29 S. Torquato, Random Heterogeneous Materials: $\mathrm{Mi}$ crostructure and Macroscopic Properties (Springer, New York, 2002).

30 D. S. McLachlan, Solid State Commun. 72, 831 (1989); D. S. McLachlan and M. B. Heaney, Phys. Rev. B 60, 12746 (1999).

31 R. W. Cohen, G. D, Cody, M. D. Coutts, and B. Abeles, Phys. Rev. B 8, 3689 (1973).

32 T. Zhu and Y. J. Wang, Phys. Rev. B 60, 11918 (1999).

33 O. V. Stogneǐ, A. V. Sitnikov, Yu. E. Kalinin, S. F. Avdeev, and M. N. Kopytin, Fiz. Tverd. Tela (st. Petersburg) 49, 158 (2007) [Phys. Solid State 49, 164 (2007)].

34 S. Barzilai, Y. Goldstein, I. Balberg, and J. S. Helman, Phys. Rev. B 23, 1809 (1981).

35 See attached Supplemental Material for fits of experimental data using the minimal EMA model of Eq. (26) with $t=2$ and $t=1$.

36 P. G. Borziak, Yu. A. Kulyupin, S. A. Nepijko, and V. G. Shamonya, Thin Solid Films 76, 359 (1981).

37 This argument is equivalent to considering a gapped radial distribution function such that $g_{2}(r)=0$ for $D \leq r \leq D_{\Delta}$ and $g_{2}(r) \neq 0$ for $r>D_{\Delta}$.

38 H. B. Michaelson, J. Appl. Phys. 48, 4729 (1977).

39 J. Robertson, J. Vac. Sci. Technol. B 18, 1785 (2000); H. Iwasaki and K. Sudoh, Jpn. J. Appl. Phys. 41, 7496 (2002).

40 A. C. Arias, L. S. Roman, T. Kugler, R. Toniolo, M. S. Meruvia, and I. A. Hummelgen, Thin Solid Films 371, 201 (2000); A. M. Serventi, D. G. Rickerby, M. C. Horrillo, and R. G. Saint-Jacques, Thin Solid Films 445, 38 (2003).

41 A. R. Kansal, S. Torquato, and F. H. Stillinger, J. Chem. Phys. 117, 8212 (2002).

42 From Eqs. 222 and (23) we recover the fully penetrable sphere limit $\phi=1-\exp (-\eta)$ for $\lambda=0$ and the fully impenetrable (hard-core) sphere limit $\phi=\eta$ for $\lambda=1$.

43 A. Trokhymchuk, I. Nezbeda, J. Jirsák, and D. Henderson, J. Chem. Phys. 123, 024501 (2005); 124, 149902(E) (2006).

44 M. A. Miller, J. Chem. Phys. 131, 066101 (2009).

45 J. J. Salacuse and G. Stell, J. Chem. Phys. 77, 3714 (1982); S. Torquato and G. Stell, J. Chem. Phys. 80, 878 (1984).

46 C. Grimaldi, M. Mionić, R. Gaal, L. Forró, and A. Magrez, Appl. Phys. Lett. 102, 223114 (2013).

47 Z. Konstantinovic, M. Garcia del Muro, M. Vaela, X. Battle, and A. Labarta, Nanotechnology 17, 4106 (2006).

48 S. B. Yuste and A. Santos, J. Chem. Phys. 101, 2355 (1994). 


\section{SUPPLEMENTAL MATERIAL FOR "THEORY OF PERCOLATION AND TUNNELING REGIMES IN NANOGRANULAR METAL FILMS"}

To evaluate the effect of varying the transport exponent $t$ in the EMA model of Sec. III, we fit the experimental conductivity data of Refs. 1, 12, 14 16, 31 34 with the EMA conductivity resulting from the solution of

$$
\frac{\left(\phi / \phi_{c}\right) g_{m}^{1 / t}}{\bar{G}^{1 / t}+g_{m}^{1 / t}}+4 \phi\left\{\left[1+\frac{\xi}{2 D} \ln \left(\frac{g_{t}+\bar{G}}{\bar{G}}\right)\right]^{3}-1\right\}=1,
$$

with transport exponent fixed at $t=2$ and $t=1$. The case $t=2$ corresponds to the approach followed in Sec. III in which $t$ is adjusted to reproduce the percolation exponent for three dimensional systems. The case $t=1$ is the EMA exponent. The resulting fits are shown in Figs. 9, 13] and the corresponding values of $\phi_{c}, \xi / D, g_{t} / g_{m}$, and $\Sigma$ are reported in Table
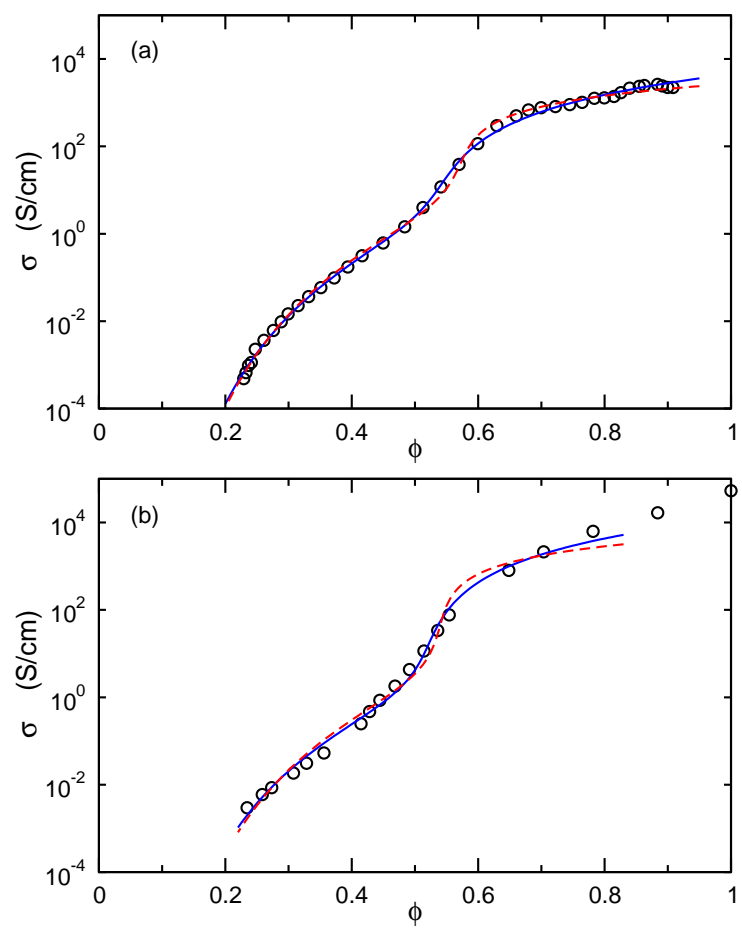

FIG. 9. (Color online) Measured conductivity $\sigma$ (open circles) as a function of Ni content for $\mathrm{Ni}_{-} \mathrm{SiO}_{2}$ granular films. Data are taken (a) from Ref. 14 and (b) from Ref. 1. Solid (dashed) lines are fitting curves from solutions of Eq. (26) with $t=2(t=1)$. 

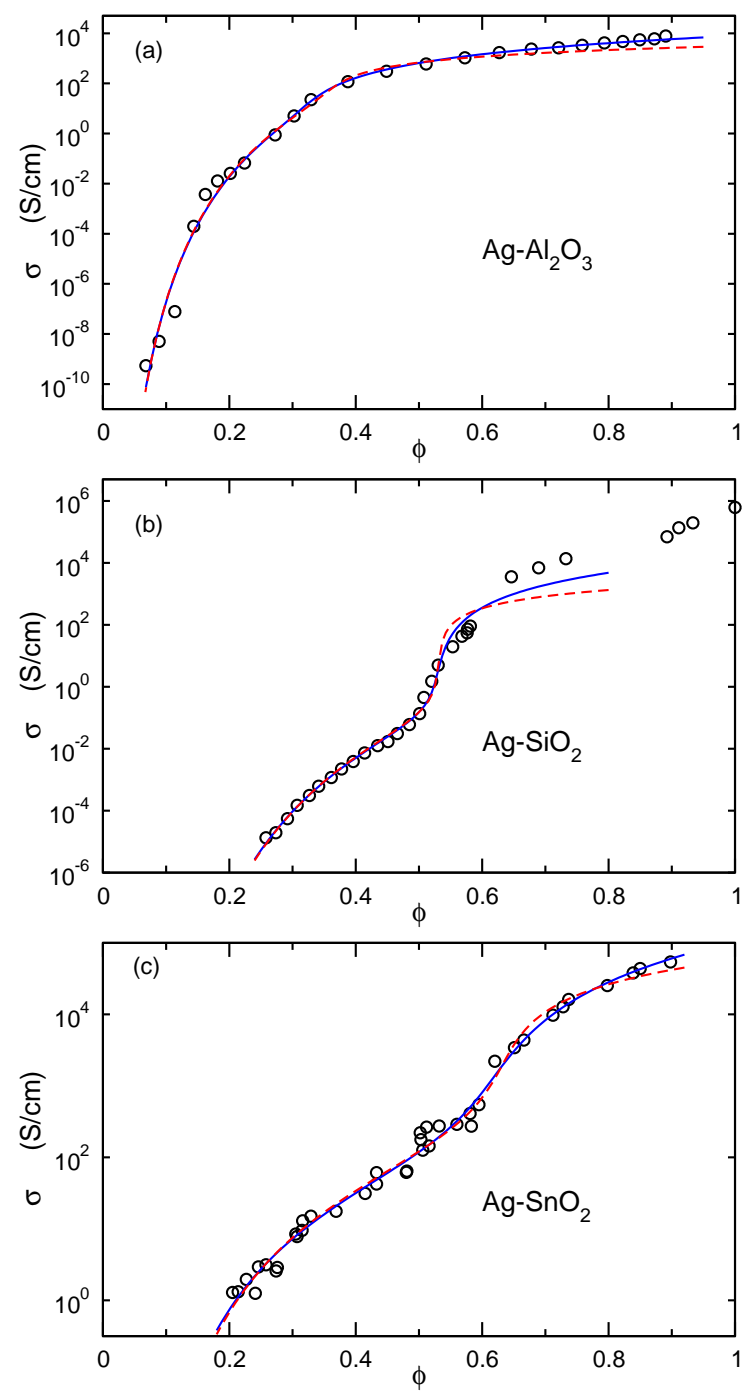

FIG. 10. (Color online) Measured conductivity $\sigma$ (open circles) as a function of $\mathrm{Ag}$ content for (a) $\mathrm{Ag}-\mathrm{Al}_{2} \mathrm{O}_{3}, \frac{16}{2}$ (b) $\mathrm{Ag}$-SiO $\mathrm{Si}_{2}, \frac{31}{,}$

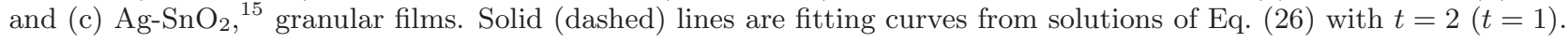

TABLE III. Values of $\phi_{c}, \xi / D, g_{t} / g_{m}$, and $\Sigma$ that best fit the measured conductivity data of Refs. 1, 12, 14 16, 31 34 obtained using the EMA semi-phenomenological model of Eq. (26) with $t=2$ and $t=1$.

\begin{tabular}{|c|c|c|c|c|c|c|c|c|}
\hline \multirow[t]{2}{*}{ Material } & \multicolumn{2}{|c|}{$\phi_{c}$} & \multicolumn{2}{|c|}{$\xi / D$} & \multicolumn{2}{|c|}{$g_{t} / g_{m}$} & \multicolumn{2}{|c|}{$\Sigma(\mathrm{S} / \mathrm{cm})$} \\
\hline & $t=2$ & $t=1$ & $t=2$ & $t=1$ & $t=2$ & $t=1$ & $t=2$ & $t=1$ \\
\hline $\mathrm{Ni}^{-\mathrm{SiO}_{2}}$ (Ref. 14) & 0.52 & 0.57 & 0.045 & 0.042 & $2.9110^{-4}$ & $1.1010^{-3}$ & $5.4810^{3}$ & $3.6310^{3}$ \\
\hline $\mathrm{Ni}^{-\mathrm{SiO}_{2}}$ (Ref. 1) & 0.51 & 0.54 & 0.052 & 0.047 & $7.8410^{-5}$ & $4.0710^{-4}$ & $1.3110^{4}$ & $5.9210^{3}$ \\
\hline $\mathrm{Ag}-\mathrm{Al}_{2} \mathrm{O}_{3}$ (Ref. 16$)$ & 0.3 & 0.36 & 0.046 & 0.044 & $2.910^{-3}$ & $1.8410^{-2}$ & $1.4610^{3}$ & $1.7810^{3}$ \\
\hline Ag- $\mathrm{SiO}_{2}$ (Ref. 31) & 0.52 & 0.53 & 0.03 & 0.03 & $6.2610^{-6}$ & $5.3210^{-5}$ & $1.7510^{4}$ & $2.6310^{3}$ \\
\hline $\mathrm{Ag}-\mathrm{SnO}_{2}$ (Ref. 15) & 0.59 & 0.64 & 0.091 & 0.085 & $4.6010^{-4}$ & $1.5210^{-3}$ & $2.1210^{5}$ & $110^{5}$ \\
\hline $\mathrm{Au}-\mathrm{Al}_{2} \mathrm{O}_{3}$ (Ref. 1 ) & 0.38 & 0.39 & 0.043 & 0.041 & $1.3510^{-7}$ & $7.1210^{-7}$ & $3.0210^{4}$ & $8.3610^{3}$ \\
\hline $\mathrm{Au}-\mathrm{SiO}_{2}$ (Ref. 31) & 0.45 & 0.45 & 0.048 & 0.046 & $7.7410^{-7}$ & $2.1310^{-6}$ & $5.1410^{4}$ & $2.4310^{4}$ \\
\hline $\mathrm{Fe}-\mathrm{Al}_{2} \mathrm{O}_{3}$ (Ref. 32) & 0.50 & 0.53 & 0.061 & 0.057 & $1.2910^{-4}$ & $5.810^{-4}$ & $1.6010^{4}$ & $5.8510^{3}$ \\
\hline $\mathrm{Fe}-\mathrm{SiO}_{2}$ (Ref. 12) & 0.41 & 0.44 & 0.078 & 0.071 & $2.8910^{-5}$ & $1.210^{-4}$ & $4.0310^{3}$ & $2.4110^{3}$ \\
\hline $\mathrm{Co}-\mathrm{Al}_{2} \mathrm{O}_{n}$ (Ref. 33) & 0.62 & 0.64 & 0.026 & 0.026 & $1.2210^{-4}$ & $1.210^{-3}$ & $2.1810^{5}$ & $3.3410^{4}$ \\
\hline Co-SiO 2 (Ref. 34) & 0.56 & 0.57 & 0.097 & 0.089 & $1.0210^{-5}$ & $6.110^{-5}$ & $1.5010^{4}$ & $3.6610^{3}$ \\
\hline
\end{tabular}



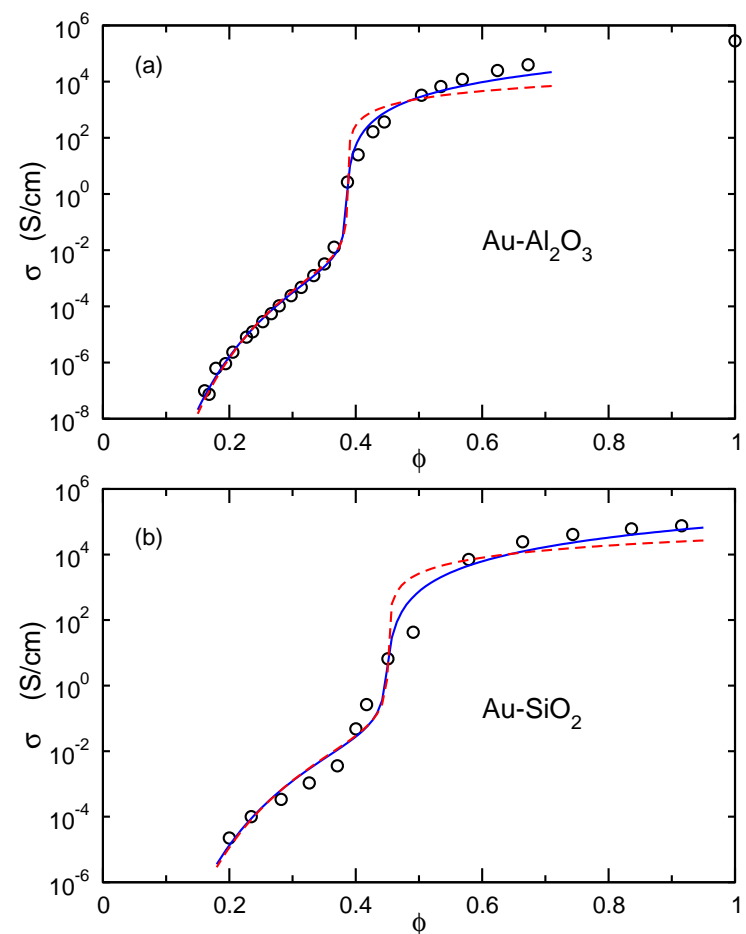

FIG. 11. (Color online) Measured conductivity $\sigma$ (open circles) as a function of $\mathrm{Au}$ content for (a) $\mathrm{Au}^{-\mathrm{Al}} \mathrm{O}_{2} \mathrm{O}_{3} \stackrel{1}{\frac{1}{2}}$ and $(\mathrm{b})$ $\mathrm{Au}-\mathrm{SiO}_{2}, \frac{31}{2}$ granular films. Solid (dashed) lines are fitting curves from solutions of Eq. (26) with $t=2(t=1)$.
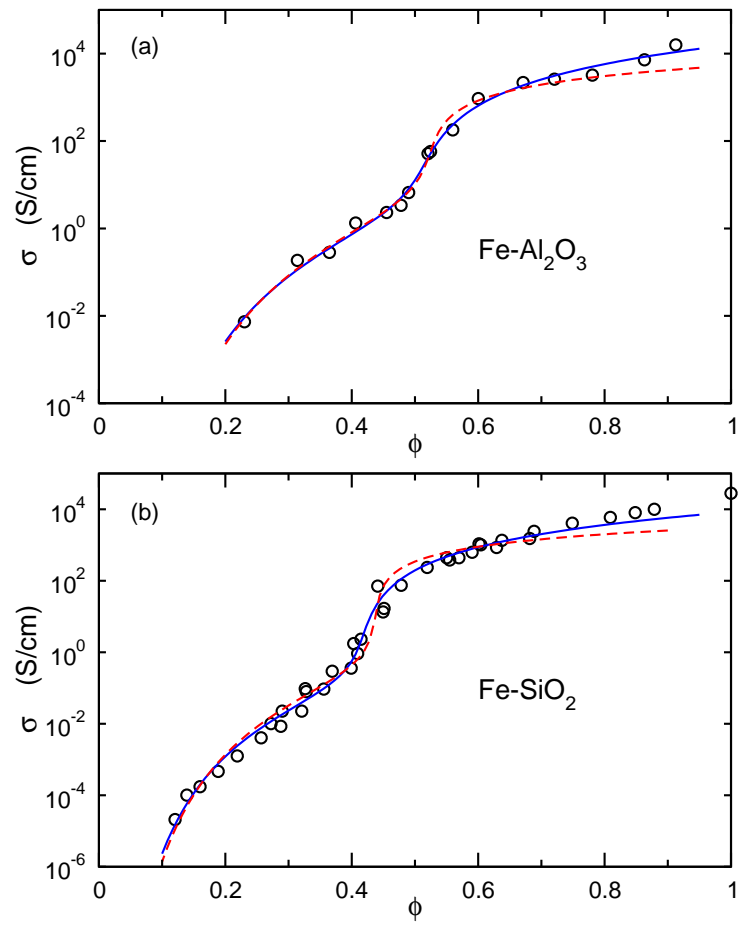

FIG. 12. (Color online) Measured conductivity $\sigma$ (open circles) as a function of Fe content for (a) $\mathrm{Fe}_{-} \mathrm{Al}_{2} \mathrm{O}_{3}, \frac{32}{,}$ and $_{(b)} \mathrm{Fe}_{-} \mathrm{SiO}_{2}, \frac{12}{,}$ granular films. Solid (dashed) lines are fitting curves from solutions of Eq. (26) with $t=2(t=1)$. 

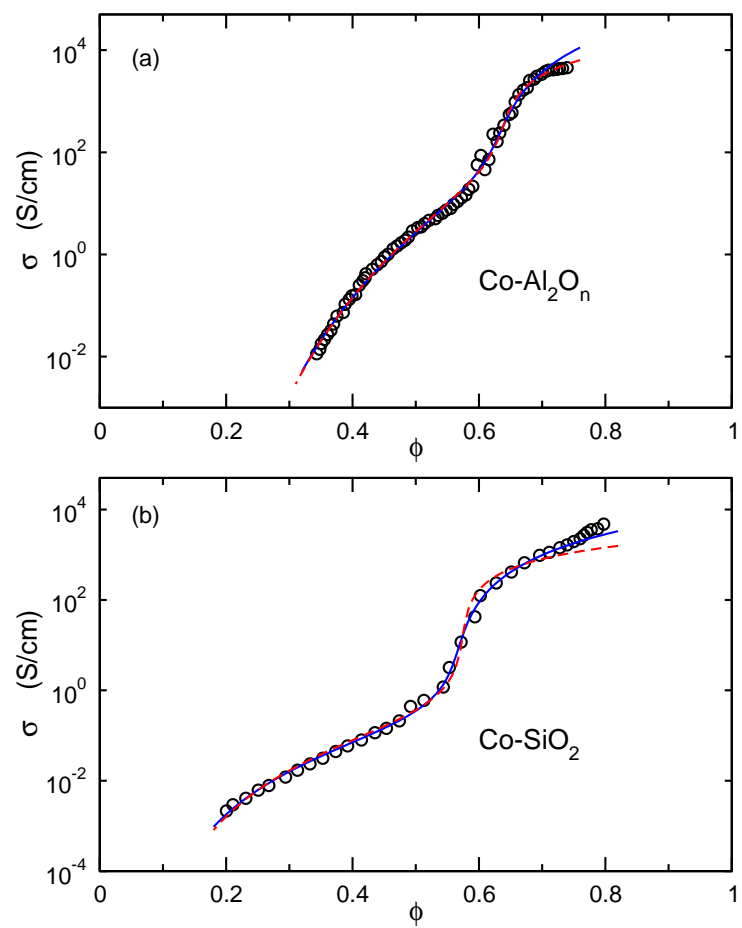

FIG. 13. (Color online) Measured conductivity $\sigma$ (open circles) as a function of Co content for (a) Co- $\mathrm{Al}_{2} \mathrm{O}_{n} \stackrel{33}{=}$ and (b)

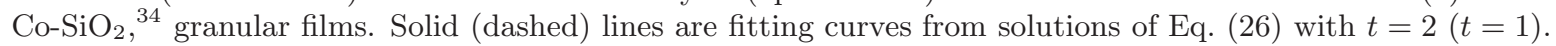

